\title{
IMAGING IN WHITE LIGHT WITH A THICK-PHASE TRANSMISSION HOLOGRAPHIC DOUBLET
}

A. BELÉNDEZ, I. PASCUAL and A. FIMIA

\section{MOTS CLÉS :}

Lentilles holographiques

Imagerie en lumière blanche

\author{
KEY WORDS : \\ Holographic Optical Elements \\ White light imaging
}

SUMMARY : An afocal two-element holographic imaging system is presented. This system can be used to work in white light. The holographic optical elements (holographic lenses) are made as thick phase holograms on silver halide sensitized gelatin (SHSG) and they present a maximum diffraction efficiency of $75 \%$. Geometrical conditions at reconstruction with coherent light and with white light are studied and a resolution test chart is imaged through the system, which shows a best resolution of 57 lines $/ \mathrm{mm}$ for coherent illumination and 15 lines $/ \mathrm{mm}$ for white light. The two-element holographic system presents a maximum diffraction efficiency of $56 \%$.
Imagerie en lumière blanche par deux hologrammes de phase épais en transmission

RÉSUMÉ : Nous présentons un système afocal à deux éléments optiques holographiques qui peut former l'image d'objets en lumière blanche. Les lentilles holographiques ont été réalisées avec des hologrammes de phase épais en gélatine bichromatée argentique (SHSG) et ils ont un rendement de diffraction maximum proche de $75 \%$. On montre les conditions géométriques de reconstruction en lumière cohérente et en lumière blanche. L'image d'une mire formée par le système montre une résolution de 57 lignes par $\mathrm{mm}$ en lumière cohérente et une résolution de 15 lignes par $\mathrm{mm}$ en lumière blanche. Le système complet a un rendement de diffraction maximum proche de $56 \%$.

\section{1. - INTRODUCTION}

An optical system can be described as a device that transforms input wave fronts into output wave fronts. According to this straightforward definition, a holographic optical element allows an analysis similar to that of conventional optical systems. From the conceptual point of view of operation, the difference between the holographic optical element (HOE) and the conventional optical element (COE) lies in the fact that the former works by diffraction and not by refraction or reflection as is the case in the latter. As a consequence of this, HOEs have some special characteristics such as great chromatic dispersion and in the case of thick holograms, strong angular dependence of the diffracted beam on the incident one. Chromatic dispersion is a fundamental limitation in the use of holograms that are reconstructed at a wavelength different than that at which they are made. The effects of dispersion are es- pecially noticeable in holograms that are reconstructed in broadband illumination.

Some of the advantages offered by holographic optical elements as opposed to conventional ones are their easy fabrication - they can be registered by what is basically a photographic process - as well as their reduced weight and volume. Taking this into account, it is possible to make optical elements of a considerable size.

High diffraction efficiency may be achieved, particulary when generating HOEs as thick phase holograms ; in this case, however, the image field and wavelength range are restricted by volume effects. Since thick holograms present these angular and wavelength selectivities, it is possible to realize a system of two holographic lenses where the longitudinal and transverse chromatic aberrations can be corrected [1], [2].

Two and three-element combinations of holographic elements producing color corrected imagery 
have been discussed by several authors. Weingartner and Rosenbruch [2] have demonstrated that the system with two elements and with three elements behaves like an achromat and an apochromat system respectively. Stone and George [3] have presented a theoretical and experimental treatment for the diffraction efficiencies of transmission HOEs and three- and two-element cascades when subject to broad spectral and field angle detuning. C. Shakher and S. Sirohi [4] have shown that a pair of thick phase holograms can be used to image extended objects in white light, but they use a blocking filter between the white light source and the transparency object.

In this paper we present an experimental realization of an afocal and partially achromatic system with two holographic optical elements. This system works well in white light. The HOEs were made as thick phase holograms. Experimental results on performance characteristics, such as the diffraction efficiency and noise, of these two holographic lenses and the resolution of this doublet as an imaging system are presented.

\section{2. - COHERENT PROCESSING SYSTEM CONFIGURATION}

An important kind of optical processing system can be characterized by a complete reliance on geometrical-optics reasoning for design. They usually have been designed for work with incoherent illumination. Such systems are comparatively simple in their construction and operation but this simplicity is sometimes achieved at the price of a limited datahandling capacity. It is also convenient to synthesize desired linear-filtering operations in the frequency domain rather than the space domain. When coherent illumination is used, the desired filtering operation can be synthesized by direct manipulation of the amplitude transmittance across the back focal plane of a transforming lens [5].

Consider the arrangement shown in figure 1. It is a typical configuration used for coherent image formation. Monochromatic light from point source $S$ is collimated by lens $L_{0}$. The object transparency is placed in plane $P_{0}$ and illuminated from the left by that collimated beam. Lens $L_{1}$ takes the Fourier transform of the transparency object. We use $P_{1}$ (focal plane of the lens $L_{1}$ ) to denote the plane that is conjugate with the source. Point source $S$, lens $L_{0}$, input plane $P_{0}$ and lens $L_{1}$ are a diffracting system. The second half of the system consists of an aperture stop located at plane $P_{1}$, to manipulate the amplitude and phase of the spectrum, a lens $L_{2}$ and an image plane $P_{2}$. The entire system therefore consists of two spectrum analysers in cascade. We have represented the $\left(x_{2}, y_{2}\right)$ coordinate system in a reflected geometry as a consequence of the reflection of the output introduced by the sequence of two Fourier transformations rather than a Fourier transformation followed by an inverse Fourier transformation [5].

This configuration satisfies the lens formula and the magnification is equal to the negative ratio of the focal lengths of the lens elements $L_{1}$ and $L_{2}$. If $f_{1}$ and $f_{2}$ are the focal lengths of the lenses $L_{1}$ and $L_{2}$ respectively, then the magnification, $m$, can be written as $m=-f_{2} / f_{1}$.

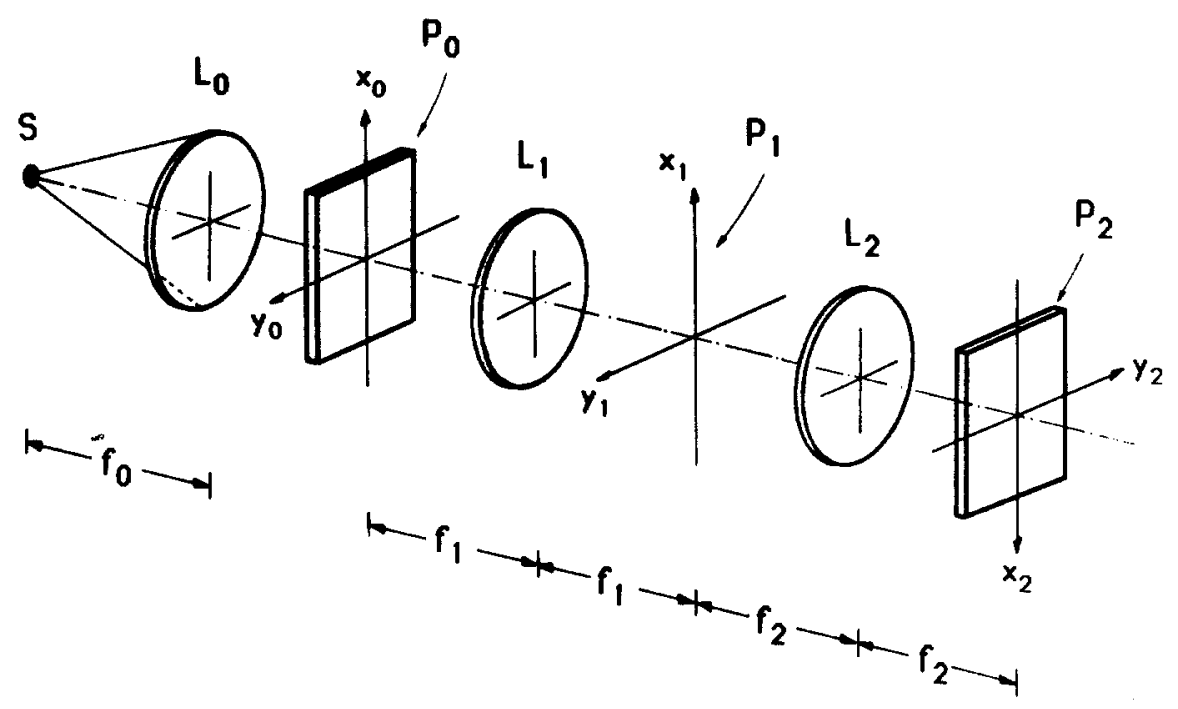

FIG. 1. - Classical configuration for a coherent processing system. $S$, monochromatic point source; $L_{0}$, collimating lens; $P_{0}$, input plane; $L_{1}$, transforming lens $; P_{1}$, frequency plane ; $L_{2}$, transforming lens; $P_{2}$, output plane. 


\section{3. - EXPERIMENTAL REALIZATION OF THE HOLOGRAPHIC SYSTEM}

Let us consider the scheme of the holographic imaging system using two thick-phase transmission holograms as shown in figure 2. The holographic optical elements used in this system were constructed in the arrangement shown in figure $3(a)$ and reconstructed in the configuration shown in figure $3(b)$. As usual, we will denote by $R, O, C$ and $I$ the sources which illuminate the object and reference light beams, and the light beams which reconstruct and form the image, respectively. We make two waves interfere with each other, one being spherical and the other collimated. To minimize the effect of astigmatism introduced by emulsion shrinkage and change in the refractive index [6], the holograms were recorded with the hologram normal along the bisector of the object-hologram-reference angle, i.e., $\alpha_{R}=-\alpha_{O}$ (see fig. 3(a)).

The image position $R_{l}$ and angle $\alpha_{I}$ are given by the expressions :

$$
\frac{1}{f}=\frac{1}{R_{I}}-\frac{1}{R_{C}}= \pm \mu\left(\frac{1}{R_{O}}-\frac{1}{R_{R}}\right)
$$

and

$$
\sin \alpha_{I}-\sin \alpha_{C}= \pm \mu\left(\sin \alpha_{O}-\sin \alpha_{R}\right)
$$

where $\mu$ denotes the ratio of the reconstruction $\left(\lambda_{C}\right)$ and recording $\left(\lambda_{R}\right)$ wavelengths and $f$ is the hologram focal length.

It is noted that for the experimental requirements of equal angles to object and reference $\left(\alpha_{R}=\right.$ $-\alpha_{O}$ ), Eq. (2) reduces to :

$$
\sin \alpha_{l}=\sin \alpha_{C} \pm 2 \mu \sin \alpha_{O} .
$$

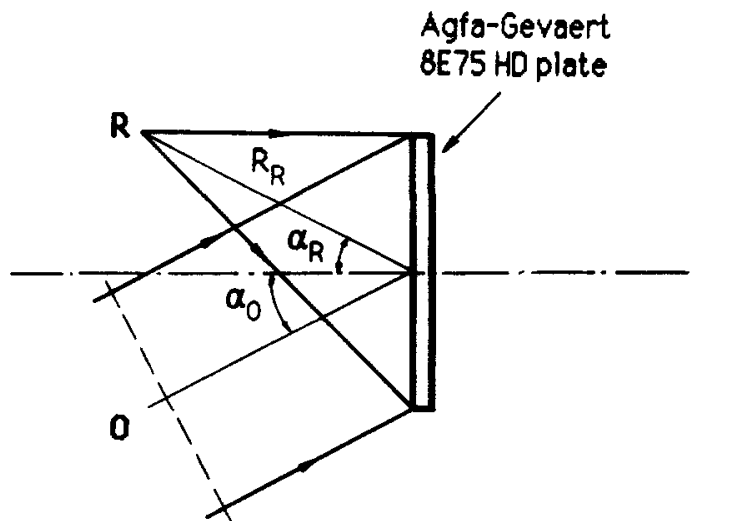

(a)

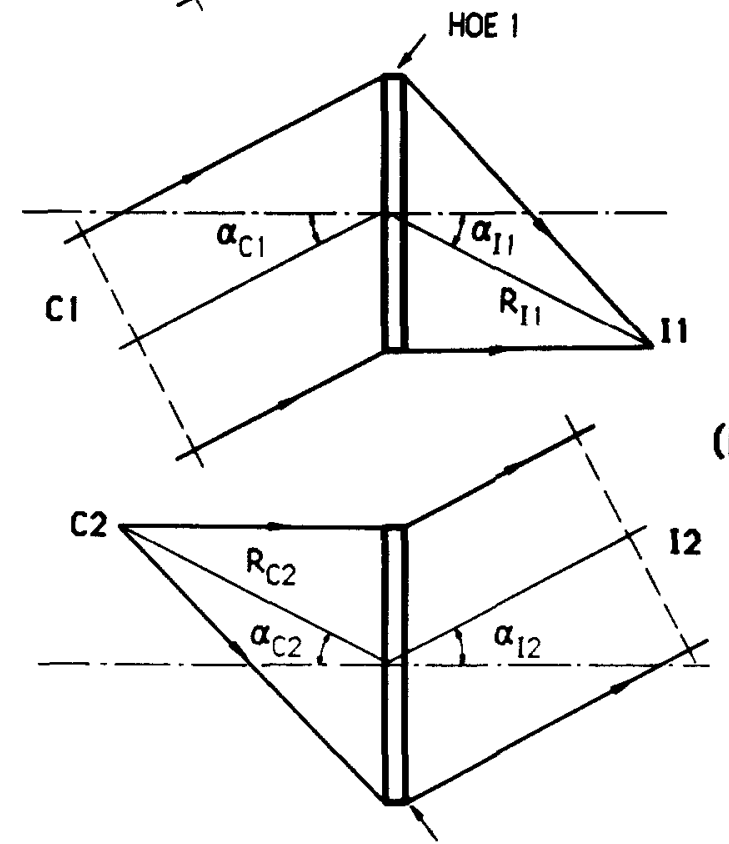

HOE 2

Fig. 3. - Hologram construction (a) and reconstruction (b) geometry.

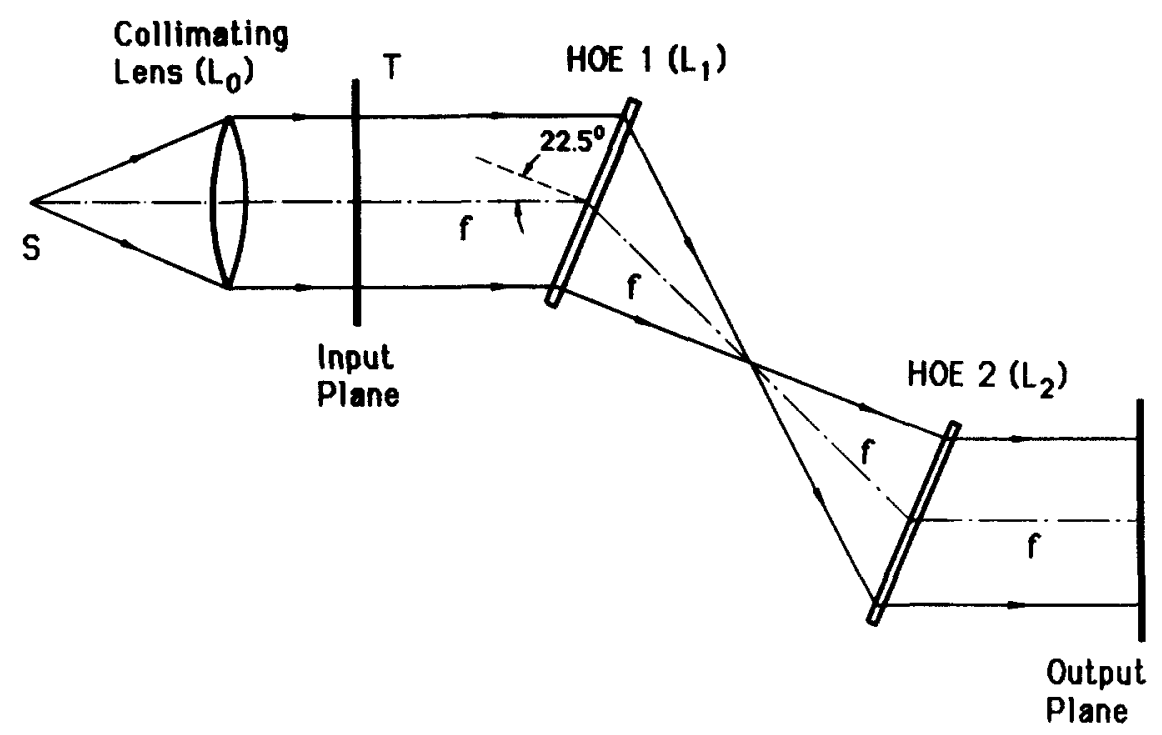

FIG. 2. - Basic scheme for the system of two holographic optical elements reconstructed with a monochromatic light collimated beam. $S$ : monochromatic light point source; $T$ : transparency object. 
For our holograms (fig. 3(b)) the expressions (1) and (3) can be written as :

$$
\begin{gathered}
R_{C 1} \rightarrow \infty, \quad R_{I 1}=R / \mu=f_{1}=f \\
\sin \alpha_{I 1}=-\sin \alpha_{C 1}=\mu \cdot \sin \theta \\
R_{C 2}=-R / \mu=-f_{2}=-f, \quad R_{I 2} \rightarrow \infty \\
\sin \alpha_{I 2}=-\sin \alpha_{C 2}=\mu \cdot \sin \theta .
\end{gathered}
$$

In the above expressions the subscripts 1 and 2 denote the first and second HOE respectively, and we have introduced the parameters $R$ and $\theta$ as $R=\left|R_{O}\right|, \theta=\left|\alpha_{R}\right|$ (fig. 3(a)).

The recording material used has been silver halide sensitized gelatin [7] in Agfa-Gevaert emulsion 8E75 $\mathrm{HD}$, using the radiation of $633 \mathrm{~nm}$ of a He-Ne laser. The HOEs present maximum diffraction efficiency near the theoretical value for Bragg angle at this wavelength.

The parameters corresponding for two HOEs is shown in table $I$.

TABLE I

Parameters for two HOEs at $633 \mathrm{~nm}$ wavelength.

\begin{tabular}{|l|c|}
\hline Exit pupil diameter $(D)$ & $55 \mathrm{~mm}$ \\
\hline Focal length $(f)$ & $275 \mathrm{~mm}$ \\
\hline Aperture $(f / D)$ & $f / 5$ \\
\hline$\theta=\left|\alpha_{R}\right|=\left|\alpha_{O}\right|$ & $22.5^{\circ}$ \\
\hline $\begin{array}{l}\text { Maximum diffraction } \\
\text { efficiency for each HOE }\end{array}$ & $75 \%$ \\
\hline $\begin{array}{l}\text { Noise } \\
\text { Maximum diffraction efficiency } \\
\text { for the holographic doublet }\end{array}$ & $<1 \%$ \\
\hline
\end{tabular}

\section{4. - HOLOGRAPHIC IMAGING SYSTEM ILLUMINATED WITH COHERENT LIGHT}

In a preliminary study, a He-Ne laser was used in reconstruction of the holograms with a pinhole of $25 \mu \mathrm{m}$ diameter (size of source $S$ ), using for the first and second HOEs $\left|\alpha_{C}\right|=\left|\alpha_{R}\right|=22.5^{\circ}$ (Bragg angles in both cases).

When we choose $\lambda_{C}=\lambda_{R}=633 \mathrm{~nm}$ and consider the angle $\alpha_{C}$ which satisfies Bragg's Law necessary to achieve maximum diffraction efficiency, the Eqs. (4)-(7) can be written as :

$$
\begin{gathered}
R_{C 1} \rightarrow \infty, \quad R_{I 1}=R=f_{1}=f \\
\sin \alpha_{I 1}=-\sin \alpha_{C 1}=-\sin \theta \\
R_{C 2}=-R=-f_{2}=-f, \quad R_{I 2} \rightarrow \infty \\
\sin \alpha_{I 2}=-\sin \alpha_{C 2}=\sin \theta .
\end{gathered}
$$

For the first HOE the image distance was the focal length of the HOE, $f=275 \mathrm{~mm}$, and for the second HOE, the reconstruction distance was also the focal length. The object for this study was the USAF three-bar resolution target. The distance between the test target and the first HOE, and between the second HOE and image plane (output plane) was the focal length (fig. 2). The resolution test chart is imaged through this system. We made a photograph of the image of the resolution test chart (fig. 4) and we found that the system can resolve approximately 57 lines $/ \mathrm{mm}$. The source used in this case had a diameter of $25 \mu \mathrm{m}$.

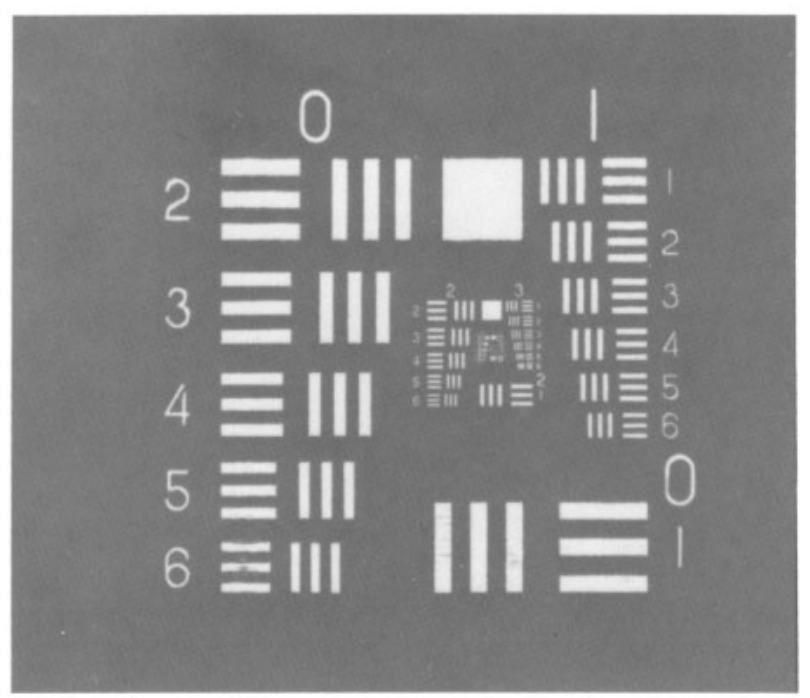

FIG. 4. - A holographic imaging system illuminated with coherent light: Photograph of the image of the resolution test chart.

\section{5. - HOLOGRAPHIC IMAGING SYSTEM ILLUMINATED WITH WHITE LIGHT}

When we reconstruct the first HOE with a collimated beam (i.e. $R_{C 1} \rightarrow \infty$ ) of white light, and with an angle $\alpha_{C 1}$, only a wavelength $\lambda_{C}$ satisfies Bragg's condition. The diffracted part of light is split spatially and spectrally and we can obtain the position of image point (determined by the coordinates $R_{I(1 k)}$ and $\alpha_{I(1 k)}$ for each wavelength $\lambda_{k}$. Combining Eqs. (1)-(3) and (4)-(7), the image position $R_{I(1 k)}$ and angle $\alpha_{I(1 k)}$ are given by the expressions :

$$
\begin{gathered}
R_{I(1 k)}=R / \mu_{k} \\
\sin \alpha_{I(1 k)}=\left(\mu-2 \mu_{k}\right) \cdot \sin \theta
\end{gathered}
$$

where $\mu=\lambda_{C} / \lambda_{R}$ is the ratio of the reconstruction wavelength $\lambda_{C}$ (for high diffraction efficiency) and construction wavelength $\lambda_{R}$, and $\mu_{k}$ denotes the wavelength shift $\lambda_{k} / \lambda_{R}$. 


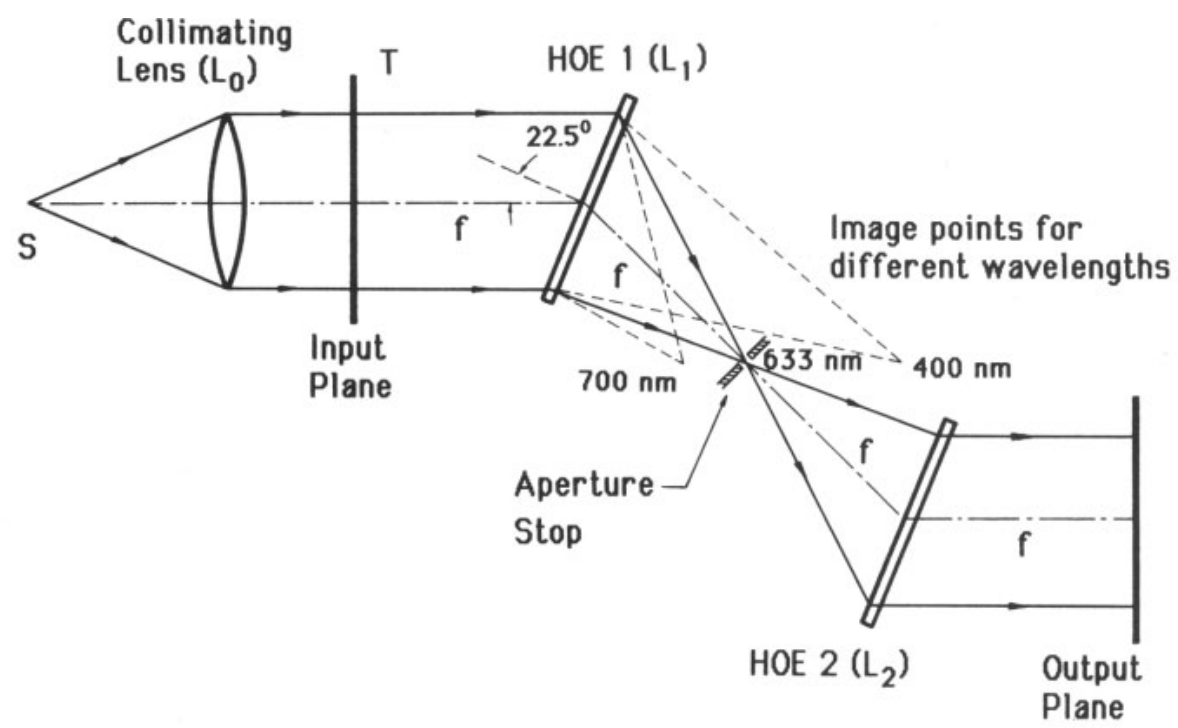

FIG. 5. - Basic scheme for the system of two holographic optical elements reconstructed with $a$ white light collimated beam. $S$ : white light point source; $T$ : transparency object.

Let's now consider a source of white light $S(55 \mathrm{~W}$ halogen lamp) with a pinhole of $2 \mathrm{~mm}$ diameter (size of source) figure 5. We reconstruct the first $\mathrm{HOE}$ with a collimated beam using $\alpha_{C 1}=22.5^{\circ}$ $\left(=-\alpha_{I 1}\right)$, which corresponds to a Bragg angle for $633 \mathrm{~nm}$ (maximum efficiency). The image distance for the first $\mathrm{HOE}$ and the reconstruction distance for the second HOE is the focal length $f=275 \mathrm{~mm}$ for $633 \mathrm{~nm}$. According to Eqs. (12) and (13) the other wavelengths focalize for different angles and distances forming the white light spectrum.

We locate an aperture stop (pinhole) at $633 \mathrm{~nm}$ image point for the first HOE. This aperture and this hologram filter white light. If the aperture stop used had a diameter of $2 \mathrm{~mm}$, the light that illuminated the second HOE had a bandwidth of $8 \mathrm{~nm}$, and when a resolution test chart is imaged through this system, it is found that this system can resolve approximately 10 lines $/ \mathrm{mm}$. Finally, when we used a pinhole of $0.5 \mathrm{~mm}$ (size of white light source) and the aperture stop used had a diameter of $2 \mathrm{~mm}$, the bandwidth was $3 \mathrm{~nm}$ and the system could resolve approximately 15 lines $/ \mathrm{mm}$. In both cases we observed the image of the test target through a $10 \times$ microscope objective and we analyzed the spectral response with a spectroradiometer S1-Topcon. Figure 6 shows the photographs of the image of the resolution test chart recorded through the holographic doublet when the source had a diameter of $2 \mathrm{~mm}$. As can be seen from the above numerical values for the resolution, when the size of the source of white light decreases the resolution is better because the bandwidth of the wavelength that illuminates the second HOE decreases too.

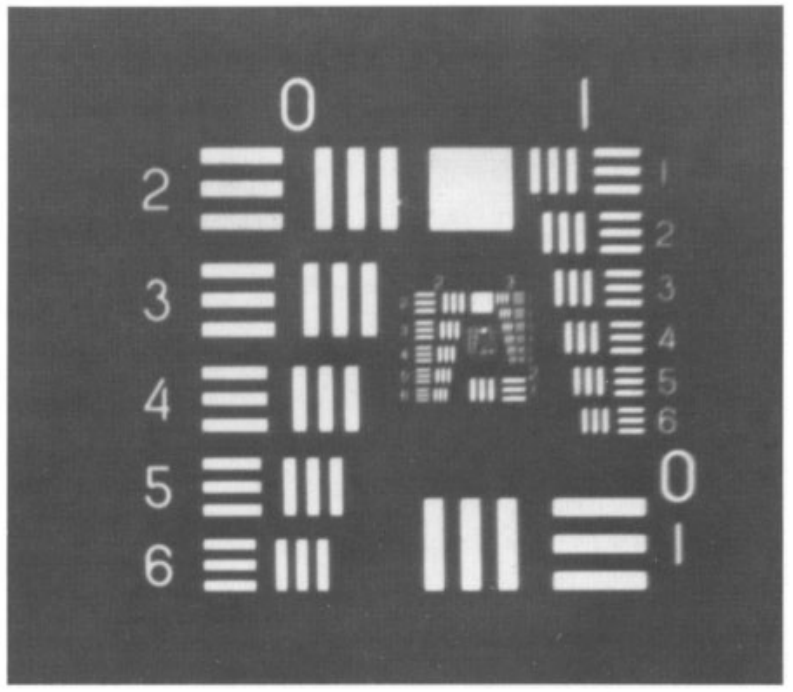

FIG. 6. - Holographic imaging system illuminated with white light. Photograph of the image of the resolution test chart when the source had a diameter of $2 \mathrm{~mm}$.

For coherent and white light illumination the distance between the test target and the first HOE was the focal length, and the analytic distance between the second HOE and image plane of the test must be the focal length too. This result agrees well with the experimental value for which the best imagery is obtained. This shows that by using white light illumination, the cascade of two off-axis elements still behaves as a coherent imaging system. 


\section{6. - CONCLUSIONS}

A holographic imaging system was described using white light illumination for which we could achieve a resolution 10 or 15 lines $/ \mathrm{mm}$ with different diameters of the source of white light and we could select the final wavelength and its bandwidth by choosing the reconstruction angle and the position and size of the aperture stop. It is important to point out that the source sizes are $2 \mathrm{~mm}$ and $0.5 \mathrm{~mm}$ diameter, and a size of aperture stop equal to $2 \mathrm{~mm}$ lead a bandwidth on the order of $8 \mathrm{~nm}$ and $3 \mathrm{~nm}$ for each size of source. These values of bandwidth are quite suitable for filtering.

The system described shows that improved broadband holographic imagery can be obtained by using two holo-lenses. Our image was quite bright due to quite high diffraction efficiency of individual holographic optical elements. Also the holographic doublet acts as a variable scale Fourier transform system.

It is evident from the above discussion that a holographic imaging system having two thick-phase HOEs can be designed and manufactured to work under white light illumination conditions with good experimental results.

\section{REFERENCES}

[1] Weingartner (I.), Rosenbruch (K. J.). - Zweistufige Achromatische Holographische Abbildungssysteme. $O p$ tik, 1980, Vol. 59, 155-166.

[2] Weingartiner (I.), Rosenbruch (K. J.). - Chromatic Correction of Two- and Three-Element Holographic Imaging Systems. Opt. Acta, 1982, Vol. 29, 519-529.

[3] Stone (T.), George (N.). - Wavelength Performance of Holographic Optical Elements. Appl. Opt., 1985, Vol. 24, 3797-3810.

[4] Shakher (C.), Sirohi (S.). - Imaging in White Light by Two Holo-Lenses. J. Optics (Paris), 1985, Vol. 16, 225227.

[5] Goodmann (J. W.). - Book « Introduction to Fourier Optics ». McGraw-Hill, New York 1968, pp. 157-169.

[6] Beléndez (A.), Pascual (I.), Fimia (A.). - Influences of Recording Materials in HOE, 1989. Proceeding SPIE, vol. 1136.

[7] Fimia (A.), Pascual (I.), Beléndez (A.). - Silver Halide Sensitized Gelatin as a Holographic Storage Medium, 1988. Proceeding SPIE, Vol. 952, 288-291.

(Manuscrit reçu le 28 mai 1989.)

\section{BULLETIN D'ABONNEMENT 1990/SUBSCRIPTION ORDER FORM 1990}

Je désire souscrire un abonnement à la :

I wish to subscribe to :

\section{Nouvelle Revue d'Optique - Journal of Optics}

1 an $/ 1$ year... $6 \mathrm{~N}^{\circ} / 6$ issues... $1156 \mathrm{~F}$.

foreign countries : 230 US \$

Nom/Name

Prénom/First name

Adresse/Address

Code Postal/Zip code

Ville/Town

Pays/Country

Spécialité/Speciality

Tél./Phone $N^{\circ}$

Bulletin à retourner, accompagné de votre règlement (libellé à l'ordre de E.S.I.), à :

Please, return this form with your payment (to E.S.I.), to.

E.S.I., B.P. 22, 41353 Vineuil FRANCE (')

(1) For foreign countries check at page 2 the address of each subscription representative per country 